\title{
THE EXISTENCE OF T-NUMBERS IN POSITIVE CHARACTERISTIC
}

\author{
TOMOHIRO OOTO
}

\begin{abstract}
As an analogue of Mahler's classification for real numbers, Bundschuh introduced a classification for Laurent series over a finite field, divided into $A, S, T, U$-numbers. It is known that each of $A, S, U$-numbers is nonempty. On the other hand, the existence of $T$-numbers is open. In this paper, we give an affirmative answer to the problem.
\end{abstract}

\section{INTRODUCTION}

From the viewpoint of Diophantine approximation, Mahler [11 introduced a classification for real numbers, divided into $A, S, T, U$-numbers. A real number is algebraic over $\mathbb{Q}$ if and only if it is an $A$-number. Two algebraically dependent transcendental real numbers are in the same class. Almost all real numbers are $S$-numbers in the sense of Lebesgue measure. For example, $e$ is $S$-number [19. Liouville numbers are $U$-numbers, for example, the real number $\sum_{n=1}^{\infty} 1 / 2^{n !}$ is $U$-number. Therefore, $e$ and $\sum_{n=1}^{\infty} 1 / 2^{n !}$ are algebraically independent. The existence of $T$-numbers had been open for thirty-six years. Schmidt 22 proved that there exist uncountably many $T$-numbers. The proof of this result is based on a nested interval construction and a generalization of the Roth's theorem by Wirsing [26]. Since Wirsing's theorem is of ineffective nature, we note that Schmidt's construction does not give explicit examples of $T$-numbers. After that, Schmidt [23] and Baker [3] investigated T-numbers in more detail. We refer the reader to [4] for Mahler's classification.

Let $p$ be a prime. Mahler [12 also introduced an analogue of Mahler's classification for $p$-adic numbers. Schlickewei [21] proved the existence of $p$-adic $T$-numbers by using a $p$-adic analogue of Schmidt's method [22]. Pejković [18] investigated $p$-adic $T$-numbers in more detail.

Let $q$ be a power of $p$. We denote by $\mathbb{F}_{q}$ the finite field of $q$ elements, $\mathbb{F}_{q}[T]$ the ring of all polynomials over $\mathbb{F}_{q}, \mathbb{F}_{q}(T)$ the field of all rational functions over $\mathbb{F}_{q}$, and $\mathbb{F}_{q}\left(\left(T^{-1}\right)\right)$ the field of all Laurent series over $\mathbb{F}_{q}$. We call an element of $\mathbb{F}_{q}\left(\left(T^{-1}\right)\right)$ an algebraic (resp. a transcendental) Laurent series if the element is algebraic (resp. transcendental) over $\mathbb{F}_{q}(T)$. Analogues to $\mathbb{Z}, \mathbb{Q}$, and $\mathbb{R}$ are $\mathbb{F}_{q}[T], \mathbb{F}_{q}(T)$, and $\mathbb{F}_{q}\left(\left(T^{-1}\right)\right)$, respectively. Bundschuh [6] introduced an analogue of Mahler's classification in $\mathbb{F}_{q}\left(\left(T^{-1}\right)\right)$. As in the real case, he divided Laurent series into $A, S, T, U$-numbers (see Section 2 for the precise definition). A fundamental question for this classification is whether or not each of the classes is nonempty. A Laurent series is algebraic over $\mathbb{F}_{q}(T)$ if and only if it is an $A$-number (see Proposition A.3). Two algebraically dependent transcendental Laurent series are in the same class (see Proposition A.4). Sprindžuk 24] proved that almost all Laurent series are $S$-numbers in the sense of Haar measure. It is easily seen that there exist $U$-numbers, for example, the Laurent series $\sum_{n=1}^{\infty} T^{-n !}$ is $U$-number. Therefore, each of the classes except for $T$-numbers is known to be nonempty. However, the existence of $T$-numbers in $\mathbb{F}_{q}\left(\left(T^{-1}\right)\right)$ is open (see [7, 25]).

2010 Mathematics Subject Classification. primary 11J82; secondary 11J61.

Key words and phrases. Diophantine approximation, Mahler's classification, positive characteristic. 
In this paper, we solve this open problem.

Theorem 1.1. There exist uncountably many T-numbers in $\mathbb{F}_{q}\left(\left(T^{-1}\right)\right)$.

We emphasize that our method allows us to construct explicit examples of $T$-numbers in $\mathbb{F}_{q}\left(\left(T^{-1}\right)\right)$. For example, we define a sequence $\left(a_{n}\right)_{n \geq 0}$ over $\mathbb{F}_{2}$ by

$$
a_{n}= \begin{cases}1 & \text { if } n=2^{4^{k} \ell} \text { for some integer } k \geq 0 \text { and odd integer } \ell \geq 1 \\ 0 & \text { otherwise }\end{cases}
$$

Then the Laurent series $\sum_{n=0}^{\infty} a_{n} T^{-n} \in \mathbb{F}_{2}\left(\left(T^{-1}\right)\right)$ is $T$-number.

In the field of Laurent series over a finite field, Mahler [14] proved that an analogue of Roth's theorem does not hold and constructed a family of explicit counterexamples. Therefore, we prove Theorem 1.1 in a different way to Schmidt's proof. Our strategy for proving Theorem 1.1 is that we construct a Laurent series with Mahler's counterexamples and show that the Laurent series is $T$-number by using a Liouville inequality.

This paper is organized as follows. In Section 2, we recall the precise definition of Mahler's classification in $\mathbb{F}_{q}\left(\left(T^{-1}\right)\right)$ and another classification which is called Koksma's classification. We also state the main results in this paper. In Section 3 , we prepare some lemmas for the proof of the main result. In Section 4, we prove the main results and Theorem 1.1. In Appendix A, we prove basic properties of Mahler's classification stated in Section 1 and 2 .

\section{Notation And Main Result}

For a Laurent series $\xi \in \mathbb{F}_{q}\left(\left(T^{-1}\right)\right) \backslash\{0\}$, we can write $\xi=\sum_{n=N}^{\infty} a_{n} T^{-n}$, where $N \in \mathbb{Z}$, $a_{n} \in \mathbb{F}_{q}$ for all integers $n \geq N$, and $a_{N} \neq 0$. We define an absolute value on $\mathbb{F}_{q}\left(\left(T^{-1}\right)\right)$ by $|0|:=0$ and $|\xi|:=q^{-N}$. The absolute value can be uniquely extended on the algebraic closure of $\mathbb{F}_{q}\left(\left(T^{-1}\right)\right)$. We continue to write $|\cdot|$ for the extended absolute value. We denote by $\left(\mathbb{F}_{q}[T]\right)[X]$ the set of all polynomials in $X$ over $\mathbb{F}_{q}[T]$. The height of a polynomial $P(X) \in\left(\mathbb{F}_{q}[T]\right)[X]$, denoted by $H(P)$, is defined to be the maximum of the absolute values of the coefficients of $P(X)$. We denote by $\left(\mathbb{F}_{q}[T]\right)[X]_{\text {min }}$ the set of all non-constant, irreducible, and primitive polynomials in $\left(\mathbb{F}_{q}[T]\right)[X]$ whose leading coefficients are monic polynomials in $T$. For an algebraic number $\alpha \in \overline{\mathbb{F}_{q}(T)}$, there exists a unique polynomial $P(X) \in\left(\mathbb{F}_{q}[T]\right)[X]_{\min }$ such that $P(\alpha)=0$. We call the polynomial $P(X)$ the minimal polynomial of $\alpha$. The height (resp. the degree) of $\alpha$, denoted by $H(\alpha)(\operatorname{resp} \cdot \operatorname{deg} \alpha)$, is defined to be the height of $P(X)$ (resp. the degree of $P(X)$ ).

Let $n \geq 1$ be an integer and $\xi$ be in $\mathbb{F}_{q}\left(\left(T^{-1}\right)\right)$. We denote by $w_{n}(\xi)$ (resp. $\left.w_{n}^{*}(\xi)\right)$ the supremum of the real numbers $w$ (resp. $w^{*}$ ) which satisfy

$$
0<|P(\xi)| \leq H(P)^{-w} \quad\left(\text { resp. } 0<|\xi-\alpha| \leq H(\alpha)^{-w^{*}-1}\right)
$$

for infinitely many $P(X) \in\left(\mathbb{F}_{q}[T]\right)[X]$ of degree at most $n$ (resp. $\alpha \in \overline{\mathbb{F}_{q}(T)}$ of degree at most $n$ ). We put

$$
w(\xi):=\limsup _{n \rightarrow \infty} \frac{w_{n}(\xi)}{n}, \quad w^{*}(\xi):=\limsup _{n \rightarrow \infty} \frac{w_{n}^{*}(\xi)}{n}
$$


We say that a Laurent series $\xi \in \mathbb{F}_{q}\left(\left(T^{-1}\right)\right)$ is an

$$
\begin{gathered}
A \text {-number if } w(\xi)=0 ; \\
S \text {-number if } 0<w(\xi)<+\infty ;
\end{gathered}
$$

T-number if $w(\xi)=+\infty$ and $w_{n}(\xi)<+\infty$ for all integers $n \geq 1$;

$U$-number if $w(\xi)=+\infty$ and $w_{n}(\xi)=+\infty$ for some integer $n \geq 1$.

This classification is called Mahler's classification. Replacing $w_{n}$ and $w$ with $w_{n}^{*}$ and $w^{*}$, we define $A^{*}, S^{*}, T^{*}$, and $U^{*}$-numbers as the above. This classification was first introduced by Bugeaud [4. Section 9] and is called Koksma's classification. $\xi$ is an A-number if and only if it is an $A^{*}$-number (see Proposition A.3). The following two statements are in [16, p.145]. If $\xi$ is an $S$-number, then it is an $S^{*}$-number. $\xi$ is a $U$-number if and only if it is a $U^{*}$-number. Therefore, we deduce that if $\xi$ is a $T^{*}$-number, then it is a $T$-number.

Let $\xi \in \mathbb{F}_{q}\left(\left(T^{-1}\right)\right)$ be a $T$-number. The type of $\xi$, denoted by $t(\xi)$, is defined to be

$$
t(\xi)=\limsup _{n \rightarrow \infty} \frac{\log w_{n}(\xi)}{\log n} .
$$

Note that we see $t(\xi) \in[1,+\infty]$ by Lemma A.1. Replacing $w_{n}$ with $w_{n}^{*}$, for $T^{*}$-number $\xi \in \mathbb{F}_{q}\left(\left(T^{-1}\right)\right)$, we define the $*$-type of $\xi$, denoted by $t^{*}(\xi)$ as the above. Note that we also see $t^{*}(\xi) \in[1,+\infty]$ by Lemma A.1.

Let $r$ be a power of $p$. We put $\alpha:=\sum_{n=1}^{\infty} T^{-r^{n}}$. Mahler [14] showed that $\alpha$ is the algebraic Laurent series of degree $r$, and satisfies $w_{1}(\alpha)=r-1$ and

$$
\alpha^{r}=\alpha-T^{-r} .
$$

Note that, in the case of $r>2$, the algebraic Laurent series $\alpha$ is the first counterexample of the Roth's theorem in $\mathbb{F}_{q}\left(\left(T^{-1}\right)\right)$, that is, $\alpha$ does not satisfy $w_{1}(\alpha)=1$.

Let $\mathbf{m}=\left(m_{j}\right)_{j \geq 0}$ be an integer sequence with $m_{0}=1, m_{j} \geq 2$ for all integers $j \geq 1$. For an integer $j \geq 0$, we put $r_{j}:=r^{m_{0} m_{1} \cdots m_{j}}$ and $\alpha_{j}(r, \mathbf{m}):=\sum_{n=1}^{\infty} T^{-r_{j}^{n}}$. We define a Laurent series $\xi(r, \mathbf{m})$ by

$$
\xi(r, \mathbf{m})=\sum_{j=0}^{\infty} \alpha_{j}(r, \mathbf{m}) .
$$

Note that since $\lim _{j \rightarrow \infty}\left|\alpha_{j}(r, \mathbf{m})\right|=0$, the Laurent series $\xi(r, \mathbf{m})$ converges.

Example. Let $p=r=2$ and $m_{j}=2$ for all $j \geq 1$. We write $\xi(r, \mathbf{m})=\sum_{n=0}^{\infty} a_{n} T^{-n}$. Then we have

$$
a_{n}= \begin{cases}1 & \text { if } n=2^{4^{k} \ell} \text { for some integer } k \geq 0 \text { and odd integer } \ell \geq 1 \\ 0 & \text { otherwise }\end{cases}
$$

Our main result of this paper is the following theorem.

Theorem 2.1. For any $r$ ans $\mathbf{m}$ defined as above, the Laurent series $\xi(r, \mathbf{m})$ are $T$ numbers and $T^{*}$-numbers.

We estimate type and $*$-type of the Laurent series $\xi(r, \mathbf{m})$.

Theorem 2.2. For any $r$ ans $\mathbf{m}$ defined as above, the Laurent series $\xi(r, \mathbf{m})$ satisfies

$$
\begin{aligned}
& \limsup _{j \rightarrow \infty}\left(2 m_{j}-1\right) \leq t^{*}(\xi(r, \mathbf{m})) \leq \limsup _{j \rightarrow \infty}\left(m_{j}+2 m_{j} m_{j+1} \cdots m_{j+p}\right), \\
& \limsup _{j \rightarrow \infty}\left(2 m_{j}-1\right) \leq t(\xi(r, \mathbf{m})) \leq \limsup _{j \rightarrow \infty}\left(2 m_{j}+2 m_{j} m_{j+1} \cdots m_{j+p}\right) .
\end{aligned}
$$


Furthermore, if $m_{j} \geq 3$ for all sufficiently large $j \geq 1$, then we have

$$
\begin{aligned}
& t^{*}(\xi(r, \mathbf{m})) \leq \limsup _{j \rightarrow \infty}\left(m_{j}+2 m_{j} m_{j+1}\right), \\
& t(\xi(r, \mathbf{m})) \leq \limsup _{j \rightarrow \infty}\left(2 m_{j}+2 m_{j} m_{j+1}\right) .
\end{aligned}
$$

In the last part of this section, we mention a problem concerning Theorem 2.2 .

Problem 2.3. For any $t \in[1, \infty]$, does there exist a $T$-number $\xi\left(\right.$ resp. $T^{*}$-number $\eta$ ) such that $t(\xi)=t\left(\operatorname{resp} . t^{*}(\eta)=t\right)$ ?

If $\limsup _{j \rightarrow \infty} m_{j}=\infty$, then we have $t(\xi(r, \mathbf{m}))=t^{*}(\xi(r, \mathbf{m}))=\infty$ by Theorem 2.2. Therefore, Theorem 2.2 gives a partial answer to Problem 2.3 in the case of $t=\infty$.

\section{Preliminaries}

Lemma 3.1. Let $n \geq 1$ be an integer and $\xi$ be in $\mathbb{F}_{q}\left(\left(T^{-1}\right)\right)$. Let $k \geq 0$ be an integer with $p^{k} \leq n<p^{k+1}$. Then we have

$$
\frac{w_{n}(\xi)}{p^{k}}-n+\frac{2}{p^{k}}-1 \leq w_{n}^{*}(\xi) \leq w_{n}(\xi)
$$

Proof. See Proposition 5.6 in [16].

The following lemma is easy consequence of Lemma 3.1 .

Lemma 3.2. For a $T$-number and $T^{*}$-number $\xi \in \mathbb{F}_{q}\left(\left(T^{-1}\right)\right)$, we have $t^{*}(\xi) \leq t(\xi)$.

The following lemma is well-known and immediately seen.

Lemma 3.3. For $P(X), Q(X) \in\left(\mathbb{F}_{q}[T]\right)[X]$, we have $H(P Q)=H(P) H(Q)$.

We recall a Liouville inequality which is Korollar 3 in [15] or Proposition 3.4 in [16].

Lemma 3.4. Let $\alpha, \beta \in \overline{\mathbb{F}_{q}(T)}$ be distinct algebraic numbers of degree $m$ and $n$, respectively. Then we have

$$
|\alpha-\beta| \geq H(\alpha)^{-n} H(\beta)^{-m}
$$

As an application of the Liouville inequality, we show the following lemma. Lemma 3.5 means that if $\xi \in \mathbb{F}_{q}\left(\left(T^{-1}\right)\right)$ has a dense (in a suitable sense) sequence of very good algebraic approximations of degree at most $d$, then we can estimate the upper bound of $w_{d}^{*}(\xi)$. Some results which are relate to the lemma are known (see e.g. [1, 2, [5, 8, 16, 17]).

Lemma 3.5. Let $\xi$ be in $\mathbb{F}_{q}\left(\left(T^{-1}\right)\right), d \geq 1$ be an integer, and $\theta, \rho, \delta$ be positive numbers. Assume that there exists a sequence of distinct terms $\left(\alpha_{j}\right)_{j \geq 1}$, and an increasing and divergent sequence of real numbers $\left(\beta_{j}\right)_{j \geq 1}$ with $\alpha_{j} \in \overline{\mathbb{F}_{q}(T)}$ of degree at most $d$ and $\beta_{j} \geq 1$ for all integers $j \geq 1$, such that

$$
\begin{gathered}
d+\delta \leq \liminf _{j \rightarrow \infty} \frac{-\log \left|\xi-\alpha_{j}\right|}{\log \beta_{j}}, \quad \limsup _{j \rightarrow \infty} \frac{-\log \left|\xi-\alpha_{j}\right|}{\log \beta_{j}} \leq d+\rho, \\
\quad \limsup _{j \rightarrow \infty} \frac{\log \beta_{j+1}}{\log \beta_{j}} \leq \theta, \quad \limsup _{j \rightarrow \infty} \frac{\log H\left(\alpha_{j}\right)}{\log \beta_{j}} \leq 1 .
\end{gathered}
$$

Then we have

$$
d+\delta-1 \leq w_{d}^{*}(\xi) \leq(d+\rho) \frac{d \theta}{\delta}-1
$$


Proof. Let $\varepsilon$ be a positive number with $\varepsilon(1+d)<\delta$. Then, by the assumption, there exists an integer $c_{0} \geq 1$ such that

$$
\beta_{j}^{-d-\rho-\varepsilon} \leq\left|\xi-\alpha_{j}\right| \leq \beta_{j}^{-d-\delta+\varepsilon}, \quad \beta_{j} \leq \beta_{j+1} \leq \beta_{j}^{\theta+\varepsilon}, \quad H\left(\alpha_{j}\right) \leq \beta_{j}^{1+\varepsilon}
$$

for all integers $j \geq c_{0}$. Since $\varepsilon$ is arbitrary small, we obtain $d+\delta-1 \leq w_{d}^{*}(\xi)$.

Let $\alpha \in \overline{\mathbb{F}_{q}(T)}$ be an algebraic number of degree at most $d$ with sufficiently large height. We define an integer $j_{0} \geq c_{0}$ by

$$
\beta_{j_{0}} \leq H(\alpha)^{\frac{d(\theta+\varepsilon)}{\delta-\varepsilon(1+d)}}<\beta_{j_{0}+1} .
$$

We first consider the case of $\alpha \neq \alpha_{j_{0}}$. Since

$$
H(\alpha)^{d}<\beta_{j_{0}+1}^{\frac{\delta-\varepsilon(1+d)}{\theta+\varepsilon}} \leq \beta_{j_{0}}^{\delta-\varepsilon(1+d)}
$$

we get

$$
\left|\alpha-\alpha_{j_{0}}\right| \geq H(\alpha)^{-d} H\left(\alpha_{j_{0}}\right)^{-d}>\beta_{j_{0}}^{-d-\delta+\varepsilon} \geq\left|\xi-\alpha_{j_{0}}\right|
$$

by Lemma 3.4. Therefore, we obtain

$$
\begin{aligned}
|\xi-\alpha| & =\left|\alpha-\alpha_{j_{0}}\right| \geq H(\alpha)^{-d} H\left(\alpha_{j_{0}}\right)^{-d} \\
& \geq H(\alpha)^{-d} \beta_{j_{0}}^{-d(1+\varepsilon)} \geq H(\alpha)^{-d-\frac{d^{2}(\theta+\varepsilon)(1+\varepsilon)}{\delta-\varepsilon(1+d)}} .
\end{aligned}
$$

We next consider the case of $\alpha=\alpha_{j_{0}}$. By the assumption, we have

$$
|\xi-\alpha| \geq \beta_{j_{0}}^{-d-\rho-\varepsilon} \geq H(\alpha)^{-(d+\rho+\varepsilon) \frac{d(\theta+\varepsilon)}{\delta-\varepsilon(1+d)}} .
$$

Since $\varepsilon$ is arbitrary small, we deduce that

$$
w_{d}^{*}(\xi) \leq \max \left(d+\frac{d^{2} \theta}{\delta}-1,(d+\rho) \frac{d \theta}{\delta}-1\right)=(d+\rho) \frac{d \theta}{\delta}-1 .
$$

\section{Proof of Main Results}

Proof of Theorem 2.1. For simplicity of notation, we put $\xi:=\xi(r, \mathbf{m})$ and $\alpha_{j}:=\alpha_{j}(r, \mathbf{m})$. For an integer $j \geq 0$, we define sequences $(a(j, n))_{n \geq 1}$ and $(b(j, n))_{n \geq 1}$ in $\mathbb{F}_{q}$ by

$$
\sum_{n=0}^{j} \alpha_{n}=\sum_{n=1}^{\infty} a(j, n) T^{-r^{n}}, \quad \sum_{n=j+1}^{\infty} \alpha_{n}=\sum_{n=1}^{\infty} b(j, n) T^{-r_{j+1}^{n}} .
$$

For integers $j \geq i \geq 0$, we put $M(i, j):=m_{i} m_{i+1} \cdots m_{j}$. For convenience, we put $M(i, j):=1$ for integers $i>j \geq 0$. Then it is easy to check that

$$
b(j, n)=\ell \bmod p,
$$

where $\ell \geq 1$ is an integer with $M(j+2, j+\ell) \mid n$ and $M(j+2, j+\ell+1) \nmid n$. For integers $j \geq 0$ and $k \geq 1$, we define algebraic Laurent series $\alpha(j, k)$ by

$$
\alpha(j, k)=\sum_{n=0}^{j} \alpha_{n}+\sum_{n=1}^{k} b(j, n) T^{-r_{j+1}^{n}} .
$$

In what follows, we estimate upper bounds of degree and height of $\alpha(j, k)$. We observe that

$$
\alpha(j, k)^{r_{j}}=\sum_{n=0}^{j} \alpha_{n}^{r_{j}}+\sum_{n=1}^{k} b(j, n) T^{-r_{j+1}^{n} r_{j}}
$$


By the equation (1), for an integer $0 \leq n \leq j$, we have

$$
\begin{aligned}
\alpha_{n}^{r_{j}} & =\left(\alpha_{n}-T^{-r_{n}}\right)^{r_{n}^{M(n+1, j)-1}}=\alpha_{n}^{r_{n}^{M(n+1, j)-1}}-T^{-r_{n}^{M(n+1, j)}} \\
& =\cdots=\alpha_{n}-\sum_{i=1}^{M(n+1, j)} T^{-r_{n}^{i}}
\end{aligned}
$$

By the definition of the sequence $(a(j, n))_{n \geq 1}$, we obtain

$$
\sum_{n=0}^{j} \alpha_{n}^{r_{j}}=\sum_{n=0}^{j} \alpha_{n}-\sum_{n=1}^{M(1, j)} a(j, n) T^{-r^{n}}
$$

Therefore, by (7), $\alpha(j, k)$ is a root of the polynomial

$$
X^{r_{j}}-X+\sum_{n=1}^{M(1, j)} a(j, n) T^{-r^{n}}+\sum_{n=1}^{k} b(j, n) T^{-r_{j+1}^{n}}-\sum_{n=1}^{k} b(j, n) T^{-r_{j+1}^{n} r_{j}} .
$$

Hence, it follows from Lemma 3.3 that $\operatorname{deg} \alpha(j, k) \leq r_{j}$ and $H(\alpha(j, k)) \leq q^{r_{j+1}^{k} r_{j}}$.

For an integer $j \geq 0$, we denote by $K_{j}$ the set of all integers $k \geq 1$ with $M(j+2, j+p) \mid$ $(k+1)$ and $M(j+2, j+p+1) \nmid(k+1)$. Note that $K_{j}$ is the infinite set. We put $K_{j}=:\left\{k_{1}<k_{2}<\ldots\right\}$. We observe that for all integers $i \geq 1$,

$$
k_{i+1}-k_{i} \leq 2 M(j+2, j+p) \text {. }
$$

We show that for all integers $i \geq 1$,

$$
\left|\xi-\alpha\left(j, k_{i}\right)\right|=q^{-r_{j+1}^{k_{i}+2}}
$$

We observe that

$$
\left|\xi-\alpha\left(j, k_{i}\right)\right|=\left|\sum_{n=k_{i}+1}^{\infty} b(j, n) T^{-r_{j+1}^{n}}\right| .
$$

By ([6), we have $b\left(j, k_{i}+1\right)=0$. Then we deduce that $M(j+2, j+2) \mid\left(k_{i}+1\right)$, which implies $M(j+2, j+2) \nmid\left(k_{i}+2\right)$. Therefore, we get $b\left(j, k_{i}+2\right)=1$. Hence, we obtain (9)).

For integers $j \geq 0$ and $k \geq 1$, we put $\beta(j, k):=q^{r_{j+1}^{k} r_{j}}$. Then, by (8) and (9), we have

$$
\frac{-\log \left|\xi-\alpha\left(j, k_{i}\right)\right|}{\log \beta\left(j, k_{i}\right)}=\frac{r_{j+1}^{2}}{r_{j}}, \quad \frac{\log \beta\left(j, k_{i+1}\right)}{\log \beta\left(j, k_{i}\right)} \leq r_{j+p}^{2}
$$

for all integers $i \geq 1$. It is trivial that $r_{j+1}^{2} / r_{j}>r_{j}$ for all integers $j \geq 0$. Applying Lemma 3.5 with $d=r_{j}, \delta=\rho=r_{j+1}^{2} / r_{j}-r_{j}$, and $\theta=r_{j+p}^{2}$, we obtain

$$
\frac{r_{j+1}^{2}}{r_{j}}-1 \leq w_{r_{j}}^{*}(\xi) \leq \frac{r_{j} r_{j+1}^{2} r_{j+p}^{2}}{r_{j+1}^{2}-r_{j}^{2}}-1
$$

for all integers $j \geq 0$. Hence, it follows that $w_{n}^{*}(\xi)<+\infty$ for all integers $n \geq 1$. We also have

$$
w^{*}(\xi) \geq \limsup _{j \rightarrow \infty} \frac{w_{r_{j}}^{*}(\xi)}{r_{j}} \geq \limsup _{j \rightarrow \infty}\left(\frac{r_{j+1}^{2}}{r_{j}^{2}}-\frac{1}{r_{j}}\right)=+\infty .
$$

Thus, the Laurent series $\xi$ is $T^{*}$-number. Therefore, by Section 2 , we deduce that $\xi$ is $T$-number. 
Proof of Theorem 2.2. Assume the notation of the proof of Theorem 2.1. By (10) and Lemma 3.2, we obtain

$$
t(\xi) \geq t^{*}(\xi) \geq \limsup _{j \rightarrow \infty} \frac{\log w_{r_{j}}^{*}(\xi)}{\log r_{j}} \geq \limsup _{j \rightarrow \infty}\left(2 m_{j}-1\right) .
$$

Since $r_{j+1}^{2} /\left(r_{j+1}^{2}-r_{j}^{2}\right) \leq 2$, we deduce that for all integers $j \geq 1$ and $r_{j-1}+1 \leq n \leq r_{j}$,

$$
\frac{\log w_{n}^{*}(\xi)}{\log n} \leq \frac{\log w_{r_{j}}^{*}(\xi)}{\log r_{j-1}} \leq m_{j}+2 m_{j} m_{j+1} \cdots m_{j+p}+\frac{\log 2}{\log r_{j-1}}
$$

Therefore, we get (2)).

By (10) and Lemma 3.1, we obtain

$$
w_{r_{j}}(\xi) \leq \frac{\left(r_{j} r_{j+1} r_{j+p}\right)^{2}}{r_{j+1}^{2}-r_{j}^{2}}+r_{j}^{2}-2 \leq 3 r_{j}^{2} r_{j+p}^{2}
$$

for all integers $j \geq 0$. Therefore, it follows that, for all integers $j \geq 1$ and $r_{j-1}+1 \leq n \leq r_{j}$,

$$
\frac{\log w_{n}(\xi)}{\log n} \leq 2 m_{j}+2 m_{j} m_{j+1} \cdots m_{j+p}+\frac{\log 3}{\log r_{j-1}}
$$

which implies (3).

Assume that $m_{j} \geq 3$ for all sufficiently large $j \geq 1$. In the same way to the proof of (9), it follows that for all integers $j \geq 0$ and $k \geq 1$,

$$
q^{-r_{j+1}^{k+2}} \leq|\xi-\alpha(j, k)| \leq q^{-r_{j+1}^{k+1}}
$$

Therefore, we have

$$
\frac{r_{j+1}}{r_{j}} \leq \frac{-\log |\xi-\alpha(j, k)|}{\log \beta(j, k)} \leq \frac{r_{j+1}^{2}}{r_{j}}, \quad \frac{\log \beta(j, k+1)}{\log \beta(j, k)}=r_{j+1}
$$

for all $j \geq 0$ and $k \geq 1$. By the assumption, we obtain $r_{j+1} / r_{j}>r_{j}$ for all sufficiently large $j \geq 1$. Applying Lemma 3.5, we have

$$
w_{r_{j}}^{*}(\xi) \leq \frac{r_{j} r_{j+1}^{3}}{r_{j+1}-r_{j}^{2}}-1
$$

for all sufficiently large $j \geq 1$. In the same way to the proof of (2) and (3), we derive (4) and (5).

Proof of Theorem 1.1. Let $r$ be a power of $p$ and $\mathbf{m}=\left(m_{j}\right)_{j \geq 0}$ be an integer sequence with $m_{0}=1, m_{j} \geq 2$ for all integers $j \geq 1$. Let $\mathbf{a}=\left(a_{j}\right)_{j \geq 0}$ be an integer sequence with $a_{j} \in\{0,1\}$ for all integers $j \geq 0$. Assume that $a_{j}=1$ for infinitely many $j \geq 0$. We put

$$
\xi_{\mathbf{a}}(r, \mathbf{m}):=\sum_{j=0}^{\infty} a_{j} \alpha_{j}(r, \mathbf{m})
$$

Then there exist $r^{\prime}$ and $\mathbf{m}^{\prime}=\left(m_{j}^{\prime}\right)_{j \geq 0}$ such that $r^{\prime}$ is a power of $p, \mathbf{m}^{\prime}=\left(m_{j}^{\prime}\right)_{j \geq 0}$ is an integer sequence with $m_{0}^{\prime}=1, m_{j}^{\prime} \geq 2$ for all integers $j \geq 1$, and $\xi_{\mathbf{a}}(r, \mathbf{m})=\xi\left(r^{\prime}, \mathbf{m}^{\prime}\right)$. Therefore, the Laurent series $\xi_{\mathbf{a}}(r, \mathbf{m})$ is $T$-number. Let $\mathbf{a}^{\prime}=\left(a_{j}^{\prime}\right)_{j \geq 0}$ be an integer sequence with $\mathbf{a} \neq \mathbf{a}^{\prime}, a_{j}^{\prime} \in\{0,1\}$ for all integers $j \geq 0$, and $a_{i}^{\prime}=1$ for infinitely many $i$. We 
define $j_{0} \geq 0$ by $a_{j}=a_{j}^{\prime}$ for all $0 \leq j<j_{0}$ and $a_{j_{0}} \neq a_{j_{0}}^{\prime}$. Then we have

$$
\begin{aligned}
\left|\xi_{\mathbf{a}}(r, \mathbf{m})-\xi_{\mathbf{a}^{\prime}}(r, \mathbf{m})\right| & =\left|\left(a_{j_{0}}-a_{j_{0}}^{\prime}\right) \alpha_{j_{0}}(r, \mathbf{m})+\sum_{j=j_{0}+1}^{\infty}\left(a_{j}-a_{j}^{\prime}\right) \alpha_{j}(r, \mathbf{m})\right| \\
& =\left|\alpha_{j_{0}}(r, \mathbf{m})\right|=q^{-r_{j_{0}}} \neq 0,
\end{aligned}
$$

which implies $\xi_{\mathbf{a}}(r, \mathbf{m}) \neq \xi_{\mathbf{a}^{\prime}}(r, \mathbf{m})$. Since there are uncountably many choices of such sequences $\mathbf{a}$, the proof is complete.

\section{APPENDIX A. BASIC PROPERTIES OF MAHLER'S CLASSIFICATION}

Lemma A.1. Let $n \geq 1$ be an integer and $\xi \in \mathbb{F}_{q}\left(\left(T^{-1}\right)\right)$ be not algebraic of degree at most $n$. Then we have $w_{n}(\xi) \geq n$ and $w_{n}^{*}(\xi) \geq(n+1) / 2$.

Proof. The former estimate follows from an analogue of Minkowski's theorem for Laurent series over a finite field [13] and the later estimates are Satz.1 of [9].

The following lemma is Theorem 5.2 in [16].

Lemma A.2. Let $n \geq 1$ be an integer and $\xi \in \mathbb{F}_{q}\left(\left(T^{-1}\right)\right)$ be algebraic of degree $d$. Then we have $w_{n}(\xi), w_{n}^{*}(\xi) \leq d-1$.

From Lemmas A.1 and A.2, we see the following proposition.

Proposition A.3. Let $\xi$ be in $\mathbb{F}_{q}\left(\left(T^{-1}\right)\right)$. Then the following conditions are equivalent:

(i) $\xi$ is an $A$-number,

(ii) $\xi$ is an $A^{*}$-number,

(iii) $\xi$ is an algebraic Laurent series.

Let $\xi$ be in $\mathbb{F}_{q}\left(\left(T^{-1}\right)\right)$ and $n, H \geq 1$ be integers. We put

$$
w_{n}(\xi, H):=\min \left\{|P(\xi)| \mid P(X) \in\left(\mathbb{F}_{q}[T]\right)[X], H(P) \leq H, \operatorname{deg}_{X} P \leq n, P(\xi) \neq 0\right\} .
$$

It is easy to check that

$$
w_{n}(\xi)=\limsup _{H \rightarrow \infty} \frac{-\log w_{n}(\xi, H)}{\log H} .
$$

Proposition A.4. Let $\xi, \eta \in \mathbb{F}_{q}\left(\left(T^{-1}\right)\right)$ be transcendental Laurent series. If $\xi$ and $\eta$ are algebraically dependent over $\mathbb{F}_{q}(T)$, then $\xi$ and $\eta$ are in the same Mahler's class.

Proof. For an integer $H \geq 1$, we take a polynomial $P(X) \in\left(\mathbb{F}_{q}[T]\right)[X]$ with $H(P) \leq$ $H, \operatorname{deg}_{X} P \leq n$, and $|P(\xi)|=w_{n}(\xi, H)$. There exists $F(X, Y) \in\left(\mathbb{F}_{q}[T]\right)[X, Y]$ which is an irreducible primitive polynomial in $X$ and $Y$ such that $F(\xi, \eta)=0$. We write

$$
F(X, Y)=\sum_{i=0}^{M} \sum_{j=0}^{N} a_{i j} X^{i} Y^{j}=\sum_{i=0}^{M} B_{i}(Y) X^{i},
$$

where $a_{i j} \in \mathbb{F}_{q}[T], B_{i}(Y) \in\left(\mathbb{F}_{q}[T]\right)[Y]$, and $B_{M}(Y) \neq 0$. Since there exists $y \in \mathbb{F}_{q}(T)$ such that $P(X)$ and $F(X, y)$ have no common root, it follows that the resultant $R(Y)=$ $\operatorname{Res}_{X}(P(X), F(X, Y))$ is non-zero polynomial in $\left(\mathbb{F}_{q}[T]\right)[Y]$. Then we obtain $\operatorname{deg}_{Y} R(Y) \leq$ $n N$ and there exists an integer $c_{1} \geq 1$ such that $H(R) \leq c_{1} H^{M}$. By the basic property of resultants (see e.g. [10, p.199-200]), there exist polynomials $g(X, Y), h(X, Y) \in$ $\left(\mathbb{F}_{q}[T]\right)[X, Y]$ and an integer $c_{2} \geq 1$ such that $R(Y)=P(X) g(X, Y)+F(X, Y) h(X, Y)$ and all of the absolute values of the coefficients of $g(X, Y)$ are less than or equal to 
$c_{2} H^{M-1}$. Then we have $R(\eta)=P(\xi) g(\xi, \eta)$ and $|g(\xi, \eta)| \leq c_{3} H^{M-1}$ for some integer $c_{3} \geq 1$. Therefore, we obtain $w_{n}(\xi) \leq M-1+M w_{n N}(\eta)$ and $w(\xi) \leq M N w(\eta)$.

We change a role of $\xi$ and $\eta$, which implies $w_{n}(\eta) \leq N-1+N w_{n M}(\xi)$ and $w(\eta) \leq$ $M N w(\xi)$. This completes the proof.

Acknowledgements. The author would like to express his gratitude to Prof. Shigeki Akiyama and Prof. Hajime Kaneko for helpful comments. The author would like to thank Prof. Yann Bugeaud for several comments. The author would like to thank the referee.

\section{REFERENCES}

[1] B. Adamczewski, Y. Bugeaud, Mesures de transcendance et aspects quantitatifs de la méthode de Thue-Siegel-Roth-Schmidt, Proc. Lond. Math. Soc. (3) 101 (2010), no. 1, 1-26 (in French).

[2] M. Amou, Approximation to certain transcendental decimal fractions by algebraic numbers, J. Number Theory 37 (1991), no. 2, 231-241.

[3] R. C. Baker, On approximation with algebraic numbers of bounded degree, Mathematika 23 (1976), no. $1,18-31$.

[4] Y. Bugeaud, Approximation by algebraic numbers, Cambridge Tracts in Mathematics, 160 Cambridge University Press, Cambridge, 2004.

[5] Y. Bugeaud, Continued fractions with low complexity: transcendence measures and quadratic approximation, Compos. Math. 148 (2012), no. 3, 718-750.

[6] P. Bundschuh, Transzendenzmasse in Körpern formaler Laurentreihen, J. reine angew. Math. 299/300 (1978), 411-432 (in German).

[7] E. Dubois, On Mahler's classification in Laurent series fields, Symposium on Diophantine Problems (Boulder, CO, 1994). Rocky Mountain J. Math. 26 (1996), no. 3, 1003-1016.

[8] A. Firicel, Rational approximations to algebraic Laurent series with coefficients in a finite field, Acta Arith. 157 (2013), no. 4, 297-322.

[9] N. Guntermann, Approximation durch algebraische Zahlen beschränkten Grades im Körper der formalen Laurentreihen, Monatsh. Math. 122 (1996), no. 4, 345-354 (in German).

[10] S. Lang, Algebra, 3rd ed., Graduate Texts in Mathematics, vol. 211 Springer, New York (2002).

[11] K. Mahler, Zur Approximation der Exponentialfunktionen und des Logarithmus, I, II, J. reine angew. Math., 166, (1932), 118-150 (in German).

[12] K. Mahler, Über eine Klasseneinteilung der p-adischen Zahlen, Mathematica Leiden 3 (1934/35), 177-185 (in German).

[13] K. Mahler, An analogue to Minkowski's geometry of numbers in a field of series, Ann. of Math. (2) 42, (1941), 488-522.

[14] K. Mahler, On a theorem of Liouville in fields of positive characteristic, Canadian J. Math. 1, (1949), 397-400.

[15] R. Müller, Algebraische Unabhängigkeit der Werte gewisser Lückenreihen in nicht-archimedisch bewerteten Körpern, Results Math., 24, (1993), no. 3-4, 288-297 (in German).

[16] T. Ooto, Quadratic approximation in $\mathbb{F}_{q}\left(\left(T^{-1}\right)\right)$, Osaka J. Math. 54 (2017), no. 1, 129-156.

[17] T. Ooto, On Diophantine exponents for the Laurent series over a finite field, J. Number Theory, $185,(2018), 349-378$.

[18] T. Pejković, On p-adic T-numbers, Publ. Math. Debrecen 82 (2013), no. 3-4, 549-567.

[19] J. Popken, Zur Transzendenz von e, Math. Z., 29, (1929), 525-541 (in German).

[20] K. F. Roth, Rational approximations to algebraic numbers, Mathematika 2 (1955), 1-20.

[21] H. P. Schlickewei, p-adic T-numbers do exist, Acta Arith. 39 (1981), no. 2, 181-191.

[22] W. M. Schmidt, T-numbers do exist, 1970 Symposia Mathematica, Vol. IV (INDAM, Rome, 1968/69), 3-26 Academic Press, London.

[23] W. M. Schmidt, Mahler's T-numbers, 1969 Number Theory Institute (Proc. Sympos. Pure Math., Vol. XX, State Univ. New York, Stony Brook, N.Y., 1969), 275-286. Amer. Math. Soc., Providence, R.I.,1971. 
[24] V. G. Sprindžuk, Mahler's problem in metric number theory, Izdat. gNauka iTehnikah, Minsk, (Russian). English translation by B. Volkmann, Translations of Mathematical Monographs, 25, American Mathematical Society, Providence, RI, 1969.

[25] D. S. Thakur, From rationality to transcendence in finite characteristic, Transcendance et irrationalité, 21-48, SMF Journ. Annu., 2012, Soc. Math. France, Paris, (2012).

[26] E. Wirsing, On approximations of algebraic numbers by algebraic numbers of bounded degree, 1969 Number Theory Institute (Proc. Sympos. Pure Math., Vol. XX, State Univ. New York, Stony Brook, N.Y., 1969), pp. 213-247. Amer. Math. Soc., Providence, R.I., 1971.

Faculty of Pure and Applied Sciences, University of Tsukuba, Tennodai 1-1-1, Tsukuba, IBARAKI, 305-8571, JAPAN

E-mail address: ooto@math.tsukuba.ac.jp 